the liver, kidneys and peripheral nerve. The primary defect has been attributed to the last enzyme in the catabolic pathway of tyrosine: fumarylacetoacetate hydrolase (FAH: E. C. 3.7.1.2). Early diagnosis and timely treatment offers an improved prognosis of the tyrosinemic patients. This treatment would require an administration of 2-(2-nitoro-4-trifluoromethylbenzoyl)-1, 3cyclohexanedione (NTBC, OrfadinÒ), a low phenylalanine and tyrosine diet, and liver transplantation.

Since several years, our laboratory carry out the diagnosis and the biological follow-up of tyrosinemic of all the own territory. In this report, we summarize the biochemical phenotype of 58 infants with HRT, focusing on the laboratory findings. The 58 infants (30 boys and 28 girls and 16 boys) came from 54 different families. The age at onset of the first symptoms of the disease varied from one week to four months, Thirty three patients died in the same month that the diagnosis was established, and twenty five are actually followed up after NTBC therapy and restriction diet. Elevated concentration of succinyl acetone in the urine, hypertyrosinemia and raised a-fetoprotein level were the most common indicators at the diagnosis. Urinalysis revealed no proteinuria, no cetonuria but sometimes glycosuria. Galactose and ferric chloride tests were negative. Positive reactions with 2, 4-dinitrophenyl hydrazine and cyanide-nitroprusside were observed occasionally. Only five urine specimens presented peculiar odour.

All patients showed hyperbilirubinemia (both direct and indirect bilirubin) and deterioration of liver function (elevated ASAT, ALAT and slightly increazed PAL and GGT).

Increased plasma ferritin and b2-microglobulin levels were seen intwenty four and five infants respectively.

However, fast plasma glucose, total cholesterol, triglycerides, albumin and proteins concentrations were either normal or slightly decreased.

HRT did not affect serum creatinine, urea, calcium, phosphorus and blood ammonia levels.

\section{0-113 PAIN ASSESSMENT IN VENTILATED AND NON-VENTILATED NEONATES IN NICUS ACROSS EUROPE: EUROPEAN PAIN AUDIT IN NEONATES (EUROPAIN SURVEY)}

R Carbajal ${ }^{1},{ }^{2} \mathrm{M}$ Eriksson, ${ }^{1} \mathrm{E}$ Courtois, ${ }^{3} \mathrm{RD}$ Andersen, ${ }^{4} \mathrm{~A}$ Avila-Alvarez, ${ }^{5} \mathrm{E}$ Boyle, ${ }^{6} \mathrm{P}$ Lago, ${ }^{7} \mathrm{~K}$ Sarafidis, ${ }^{8} \mathrm{~S}$ Simons, ${ }^{9} \mathrm{~T}$ Pölkki, ${ }^{10} \mathrm{ML}$ IImoja, ${ }^{11} \mathrm{~B}$ Van Overmeire, ${ }^{12} \mathrm{~A}$ Berger, ${ }^{13} \mathrm{~T}$ Papadouri, ${ }^{14} \mathrm{M}$ Schroth, ${ }^{15} \mathrm{R}$ Tameliene, ${ }^{16} \mathrm{~S}$ Attard Montalto, ${ }^{17} \mathrm{~A}$ Dobrzanska, ${ }^{18} \mathrm{C}$ Matos, ${ }^{19} \mathrm{E}$ Europain Study group, ${ }^{20} \mathrm{~L}$ Bergqvist, ${ }^{20} \mathrm{H}$ Lagercrantz, ${ }^{21} \mathrm{KJS}$ Anand. ${ }^{1}$ Emergency Department, Hôpital Armand-Trousseau, Paris, France, ${ }^{2}$ Centre for health care sciences, Örebro University Hospital, Örebro, Sweden; ${ }^{3}$ NICU, Telemark Hospital, Skien, Norway; ${ }^{4}$ NICU, Complexo Hospitalario Universitario de a Coruña, a Coruña, Spain; ${ }^{5} \mathrm{NICU}$, University of Leicester, Leicester, UK; ${ }^{6}$ NICU, University of Padova, Padova, Italy; ${ }^{7}$ NICU, Aristotle University of Thessaloniki, Thessaloniki, Greece; ${ }^{8}$ NICU, Erasmus MC Sophia Children's Hospital, Rotterdam, Netherlands; ${ }^{9} \mathrm{NICU}$, Institute of Health Sciences University of Oulu, Oulu, Finland; ${ }^{10}$ NICU, Tallinn Children's Hospital, Tallinn, Estonia; ${ }^{11}$ NICU, Erasme Hospital, Bruxelles, Belgium; ${ }^{12}$ NICU, Univ. Klinik F. Kinder and Jugendheilkunde, Vienna, Austria; ${ }^{13}$ NICU, Arch. Makarios Hospital, Nicosia, Cyprus; ${ }^{14}$ NICU, Cnopf'sche Kinderklinik, Nuremberg, Germany; ${ }^{15}$ NICU, Perinatal Center, Kaunas, Lithuania; ${ }^{16}$ NICU, Mater Dei Hospital, Msida, Malta; ${ }^{17}$ NICU, Children's Memorial Health Institute, Warsaw, Poland; ${ }^{18}$ NICU, Maternidade Dr Alfredo Da Costa, Lisboa, Portugal; ${ }^{19}$ Europain Study Group, Europain Study Group, Paris, France; ${ }^{20}$ NICU, Karolinska University Hospital, Stockholm, Sweden; ${ }^{21}$ Department of Pediatrics Critical Care Medecine Division, University of Tennessee Health Science Center, Memphis, USA

\subsection{6/archdischild-2014-307384.180}

Background Neonates undergo many painful procedures during their NICU stay. These may include tracheal intubation/ventilation, skin-breaking procedures, drainage/suctioning of body orifices or cavities. Inherent subjectivity and difficulties of neonatal

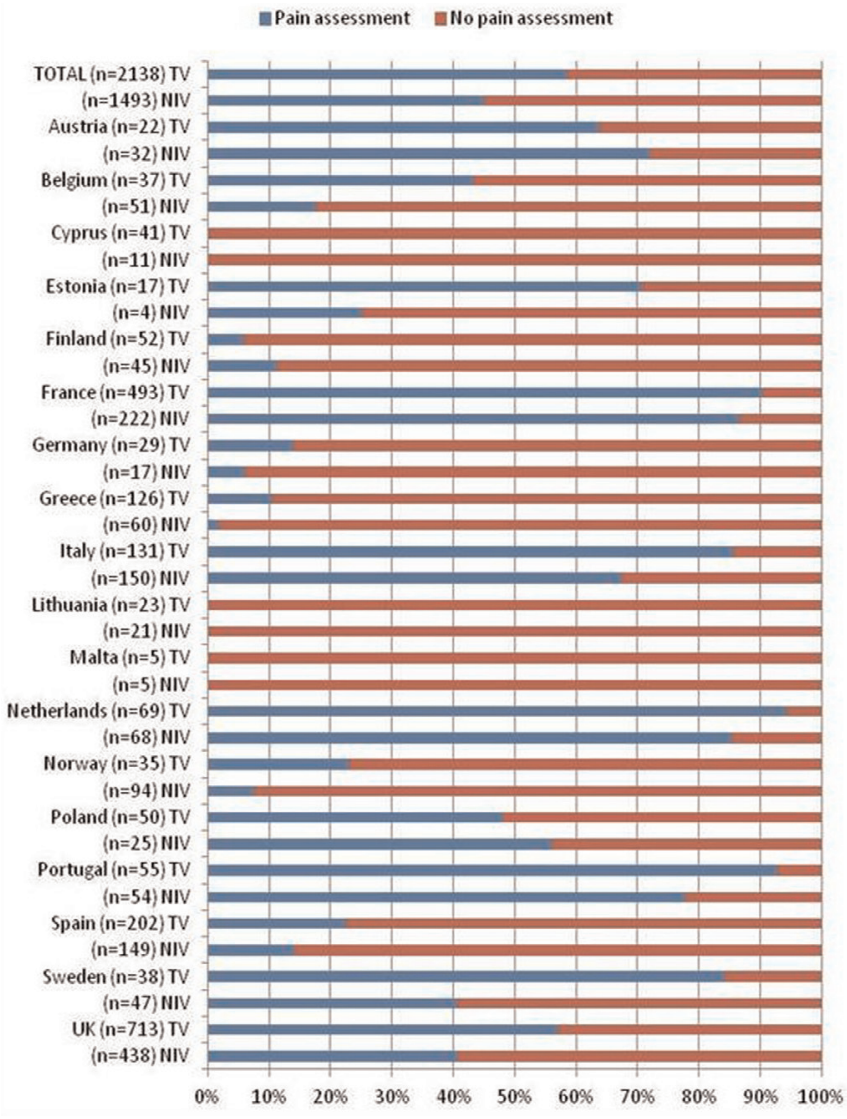

Abstract 0-113 Figure 1 Frequency of pain assessment in tracheal ventilated (TV) neonates and non invasive ventilated (NIV) neonates admitted to NICUs in 18 European countries

pain assessment contribute to a wide variety of assessment tools and clinical practices. To date, these practices have been not studied at a large scale.

Objective To determine current clinical practices for neonatal pain assessment in NICUs across Europe.

Methods An epidemiological observational study on bedside pain assessment practices collected data for all neonates in participating NICUs until infants left the unit (discharge, death, transfer to another hospital) or for 28 days. Data collection occurred via an online database for 1 month at each NICU. All neonates up to a gestational age of 44 weeks were included.

Results From October 2012 to June 2013, 243 NICUs from 18 European countries collected pain assessment data in 6680 neonates. Of these, 2142 received tracheal ventilation (TV), 1496 non-invasive ventilation (NIV) and 3042 only spontaneous ventilation (SV). The median (IQR) gestational age of TV, NIV and SV neonates were 32.1 (28.1-37.4), 33.6 (31.0-36.6) and 37.9 (35.0-39.9), respectively ( $<<0.001$ ). Overall, $58.5 \%$ of TV neonates, $45.0 \%$ of NIV neonates and $30.4 \%$ of SV neonates received bedside pain assessments $(\mathrm{p}<0.001)$. Fig. shows pain assessments by country.

Conclusions Over half (58.5\%) of TV neonates and less than half $(45.0 \%)$ of NIV neonates had pain assessments performed in European NICUs. Wide variations in the rates of pain assessment exist among countries and an important improvement seems necessary. 